\title{
One-Dimensional Tasaki-Hubbard Model in Paramagnetic Limit
}

\author{
V. BAlihA ${ }^{a}$, J. RICHTER ${ }^{b}$ AND O. DERZHKO ${ }^{a, c, b, d *}$ \\ ${ }^{a}$ Institute for Condensed Matter Physics, National Academy of Sciences of Ukraine, \\ Svientsitskii Str. 1, 79011 L'viv, Ukraine \\ ${ }^{b}$ Institut für Theoretische Physik, Otto-von-Guericke-Universität Magdeburg, \\ P.O. Box 4120, 39016 Magdeburg, Germany \\ ${ }^{c}$ Department for Theoretical Physics, Ivan Franko National University of L'viv, \\ Drahomanov Str. 12, 79005 L'viv, Ukraine \\ ${ }^{d}$ Abdus Salam International Centre for Theoretical Physics, \\ Strada Costiera 11, 34151 Trieste, Italy
}

(Received May 5, 2016)

The one-dimensional Tasaki-Hubbard model belongs to the class of flat-band ferromagnets. By introducing an infinitesimally small external magnetic field, we examine the properties of the model in the paramagnetic regime. We compare and contrast them to the properties of the conventional Curie paramagnet.

DOI: 10.12693/APhysPolA.132.1256

PACS/topics: flat-band Hubbard model, paramagnetism

\section{Introduction}

In $1992 \mathrm{H}$. Tasaki considered the standard (repulsive) one-orbital Hubbard model with the Hamiltonian

$$
\begin{aligned}
& H=\sum_{\sigma=\uparrow, \downarrow} H_{0, \sigma}+H_{U}, \\
& H_{0, \sigma}=\sum_{(i j)} t_{i j}\left(c_{i, \sigma}^{\dagger} c_{j, \sigma}+c_{j, \sigma}^{\dagger} c_{i, \sigma}\right), t_{i j}>0, \\
& H_{U}=U \sum_{i} n_{i, \uparrow} n_{i, \downarrow}, U>0
\end{aligned}
$$

on the so-called decorated lattices which support a completely dispersionless (flat) lowest-energy one-electron band [1]. In one dimension the introduced lattice is also known as the sawtooth chain or the $\Delta$-chain with a special relation between the hopping integrals along the straight line $t_{1}$ and the zigzag path $t_{2}: t_{2}=\sqrt{2} t_{1}>0$, see Fig. 1. It is well known [1] that the ground state of the model is the fully polarized (i.e., saturated) ferromagnetic state, i.e., $\left\langle\boldsymbol{S}^{2}\right\rangle_{n, \mathcal{N}}=(n / 2)[(n / 2)+1]$, if the number of electrons $n$ equals $\mathcal{N}=N / 2$ or $\mathcal{N}-1$. For smaller numbers of electrons, $1<n<\mathcal{N}-1$, we have $0<\left\langle\boldsymbol{S}^{2}\right\rangle_{n, \mathcal{N}}<(n / 2)[(n / 2)+1]$ (nonsaturated fer-

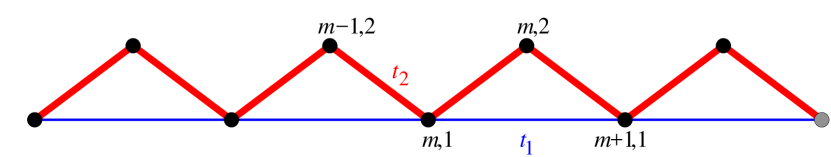

Fig. 1. One-dimensional Tasaki lattice. Here $t_{2}=$ $\sqrt{2} t_{1}>0$.

*corresponding author; e-mail: derzhko@icmp.lviv.ua romagnetism). Furthermore, $\lim _{N \rightarrow \infty}\left\langle\boldsymbol{S}^{2}\right\rangle_{n, \mathcal{N}} / n^{2}=0$, if $n / N<1 / 2$. In other words, thermodynamically large systems exhibit ground-state ferromagnetism for $n / N=1 / 2$ but are paramagnetic for $0<n / N<1 / 2$.

The Mielke-Tasaki mechanism for the ground-state ferromagnetism can be explained as follows [1-5]. $\mathcal{N}$ states from the lowest-energy flat band with the energy $\varepsilon_{1}=-2 t_{1}$ can be visualized as localized within a Vshaped part of the chain (trapping cell), i.e., the flat-band states are given by $l_{m, \sigma}^{\dagger}|0\rangle, l_{m, \sigma}^{\dagger}=c_{m-1,2, \sigma}^{\dagger}-\sqrt{2} c_{m, 1, \sigma}^{\dagger}+$ $c_{m, 2, \sigma}^{\dagger}, m=1, \ldots, \mathcal{N}$, see Fig. 1. Neighboring traps have common sites (the sites $m, 2, m=1, \ldots, \mathcal{N}$, see Fig. 1). The ground states for $1<n<\mathcal{N}-1$ electrons consist of sets of independent clusters, where each cluster is built by connected occupied traps with electrons in the symmetric spin state (ferromagnetic cluster). The many-electron ground-state states of $H$ (1) constructed this way do not feel the Hubbard repulsion $U>0$ and belong at the same time to the ground-state manifold of the noninteracting system with the energy $n \varepsilon_{1}$. For $n=\mathcal{N}$ and $n=\mathcal{N}-1$ no isolated clusters exist, i.e., only a single ferromagnetic cluster can be constructed. Therefore the ground state is ferromagnetic with the maximal value of $\left\langle\boldsymbol{S}^{2}\right\rangle_{n, \mathcal{N}}$. For smaller $1<n<\mathcal{N}-1$ isolated clusters can appear and $\left\langle\boldsymbol{S}^{2}\right\rangle_{n, \mathcal{N}}$ is less than its maximal value $S_{\max }^{2}=(n / 2)[(n / 2)+1]$.

Furthermore, one can calculate the number of the ground states, i.e., the ground-state degeneracy [5-7]. By mapping the ground states of $n<\mathcal{N}$ electrons of the $N$-site Tasaki chain onto the spatial configurations of $n$ hard dimers on a $2 \mathcal{N}$-site simple chain [6] one can find the ground-state degeneracy $g_{\mathcal{N}}(n)$ : 


$$
g_{\mathcal{N}}(n)=\frac{2 \mathcal{N}}{2 \mathcal{N}-n} \mathcal{C}_{2 \mathcal{N}-n}^{n}
$$

Bearing in mind that the canonical partition function of $n$ electrons on the Tasaki chain of volume $\mathcal{N}=N / 2$ cells is $Z(T, n, \mathcal{N})=g_{\mathcal{N}}(n) \mathrm{e}^{-n \varepsilon_{1} / T}$, we immediately get from Eq. (2) the Helmholtz free energy $F(T, n, \mathcal{N})=$ $-T \ln Z(T, n, \mathcal{N})$

$$
F(T, n, \mathcal{N})=-\mathcal{N} T \ln \frac{(2-p)^{2-p}}{p^{p}(2-2 p)^{2-2 p}}+n \varepsilon_{1},
$$

where $p=n / \mathcal{N}$ is the electron density. Alternatively, one can calculate the grand-canonical partition function $[6,7]$ :

$$
\Xi(T, \mu, \mathcal{N})=\left(\frac{1}{2}+\sqrt{\frac{1}{4}+\mathrm{e}^{\frac{\mu-\varepsilon_{1}}{T}}}\right)^{2 \mathcal{N}}
$$

and the grand thermodynamical potential

$$
\Omega(T, \mu, \mathcal{N})=-2 \mathcal{N} T \ln \left(\frac{1}{2}+\sqrt{\frac{1}{4}+\mathrm{e}^{\frac{\mu-\varepsilon_{1}}{T}}}\right) .
$$

The thermodynamic functions in Eq. (3) and Eq. (5) are related by the Legendre transformation $F=\Omega+\mu n$ after eliminating $\mu$ in the right-hand side (r.h.s.) of this equation using the relation $n=-\partial \Omega / \partial \mu$. Formulae (3) or (5) give the contribution of the highly degenerate groundstate manifold to thermodynamics of the considered Hubbard model. This contribution dominates for $0 \leq p \leq 1$ or for $\mu \approx \varepsilon_{1}$ in the low-temperature regime [5-7].

In the present paper, we wish to extend the previous investigations $[6,7]$ switching on an infinitesimally small external magnetic field $h>0^{\dagger}$, i.e., the field is nonzero but it presents the smallest energy scale in the problem. We are interested in the case $1 \leq n<\mathcal{N}-1$, that is the case which yields paramagnetism in the thermodynamic limit $n \rightarrow \infty, \mathcal{N} \rightarrow \infty, 0<p=n / \mathcal{N}<1$. Our aim is to study the peculiarities of the one-dimensional TasakiHubbard paramagnet compared to the conventional spin$1 / 2$ Curie paramagnet. These peculiarities arise (i) from the degeneracy of the ground-state manifold, (ii) from the larger size of the individual magnetic moments (which are proportional to the size of the isolated ferromagnetic clusters), and (iii) from the distribution of the size of the ferromagnetic clusters. For completeness, we give here the Helmholtz free energy of the spin- $1 / 2$ Curie paramagnet

$$
f_{\mathrm{C}}(T, h)=-T \ln \frac{\sinh \frac{h}{T}}{\sinh \frac{h}{2 T}}=-T \ln \left(2 \cosh \frac{h}{2 T}\right) .
$$

To this end, we consider finite chains consisting of $N=$ 12, 16, 20, 24 sites. After characterizing the ground-state manifold for various numbers of electrons $n=1, \ldots, N / 2$, we calculate the canonical partition functions and thermodynamic quantities. We compare the results with the case $h=0$, discuss the $1 / N \rightarrow 0$ limit, and contrast the results for the Tasaki-Hubbard and Curie paramagnets.

${ }^{\dagger}$ Here $h=g \mu_{\mathrm{B}} H$, where $H[\mathrm{~T}]$ is the magnetic field, $\mu_{\mathrm{B}} \approx$ $0.67171 \mathrm{~K} / \mathrm{T}$ is the Bohr magneton, and $g$ is the electron $g$-factor.
The discussion of finite chains allows also to check the obtained analytical results (obtained by taking into account only the flat-band cluster states) by comparing them with exact-diagonalization data for the full Hubbard model (i.e., taking into account all eigenstates) for chains of $N=16$ sites (finite $U$ ) and $N=24$ sites $(U \rightarrow \infty)$.

\section{Results}

In what follows we consider the canonical description for finite chains with $\mathcal{N}=6,8,10,12$ trapping cells. Note that in previous papers [5-7] on model (1) the focus was on grand-canonical description. In the presence of magnetic field the formula for $Z(T, n, \mathcal{N})$ must be modified. Consider, for example, the case $n=4$ and $\mathcal{N}=6$. According to Eq. (2) for $h=0$ we get $g_{6}(4)=105$, and therefore $Z(T, 4,6)=105 \mathrm{e}^{-4 \varepsilon_{1} / T}$. The ground-state degeneracy 105 comes from 6 spatial single-cluster configurations (each spatial configuration has degeneracy 5), 6 spatial configurations " 3 -site cluster +1 -site cluster" (each spatial configuration has degeneracy 8), and 3 spatial configurations "2-site cluster +2 -site cluster" (each spatial configuration has degeneracy 9). If $h>0$ the mentioned states have different energies resulting in the new formula for $Z(T, h, 4,6)$ :

$$
\begin{aligned}
& Z(T, h, 4,6)=\mathrm{e}^{-\frac{4 \varepsilon_{1}}{T}} \\
& \quad \times\left[6\left(\mathrm{e}^{\frac{2 h}{T}}+\mathrm{e}^{\frac{h}{T}}+1+\mathrm{e}^{-\frac{h}{T}}+\mathrm{e}^{-\frac{2 h}{T}}\right)\right. \\
& \quad+6\left(\mathrm{e}^{\frac{3 h}{2 T}}+\mathrm{e}^{\frac{h}{2 T}}+\mathrm{e}^{-\frac{h}{2 T}}+\mathrm{e}^{-\frac{3 h}{2 T}}\right)\left(\mathrm{e}^{\frac{h}{2 T}}+\mathrm{e}^{-\frac{h}{2 T}}\right) \\
& \left.\quad+3\left(\mathrm{e}^{\frac{h}{T}}+1+\mathrm{e}^{-\frac{h}{T}}\right)^{2}\right] .
\end{aligned}
$$

The calculation of the Helmholtz free energy per electron $f_{\mathrm{TH}}(T, h, p)=-T \ln Z(T, h, n, \mathcal{N}) / n$ and of other thermodynamic quantities is straightforward now and can be done using a software for analytical calculations.

Our results are reported in Figs. 2-6. The temperature dependence of the entropy and of the specific heat are presented in Figs. 2 and 3, respectively. For zero field, $h=0$, we know $[5-7]$ that the huge groundstate degeneracy leads to a nonzero residual entropy, i.e., $S(T, h=0, n, \mathcal{N}) / n=\left[\ln g_{\mathcal{N}}(n)\right] / n \neq 0$, as well as a vanishing specific heat, i.e., $C(T, h=0, n, \mathcal{N})=0$. By switching on $h$ the degeneracy of the ground-state manifold is partly lifted, however, the degeneracy remains huge such that a nonzero residual entropy (although reduced) survives, see Fig. 2. Trivially, for the ordinary Curie paramagnet the ground state at $h>0$ is the single ferromagnetic state, i.e., there is no residual entropy. The finite-size dependence sketched in the lower part of Fig. 2 gives evidence that the residual entropy is present for $N \rightarrow \infty$.

The specific heat at nonzero $h$ shows already a nontrivial dependence on temperature and agrees with exactdiagonalization data for model (1) at low temperatures, see Fig. 3. Moreover, finite size-effects are small (results for $\mathcal{N}=6,8,10,12$ almost coincide). As $p$ increases, deviations from the Curie-paramagnet case become more 

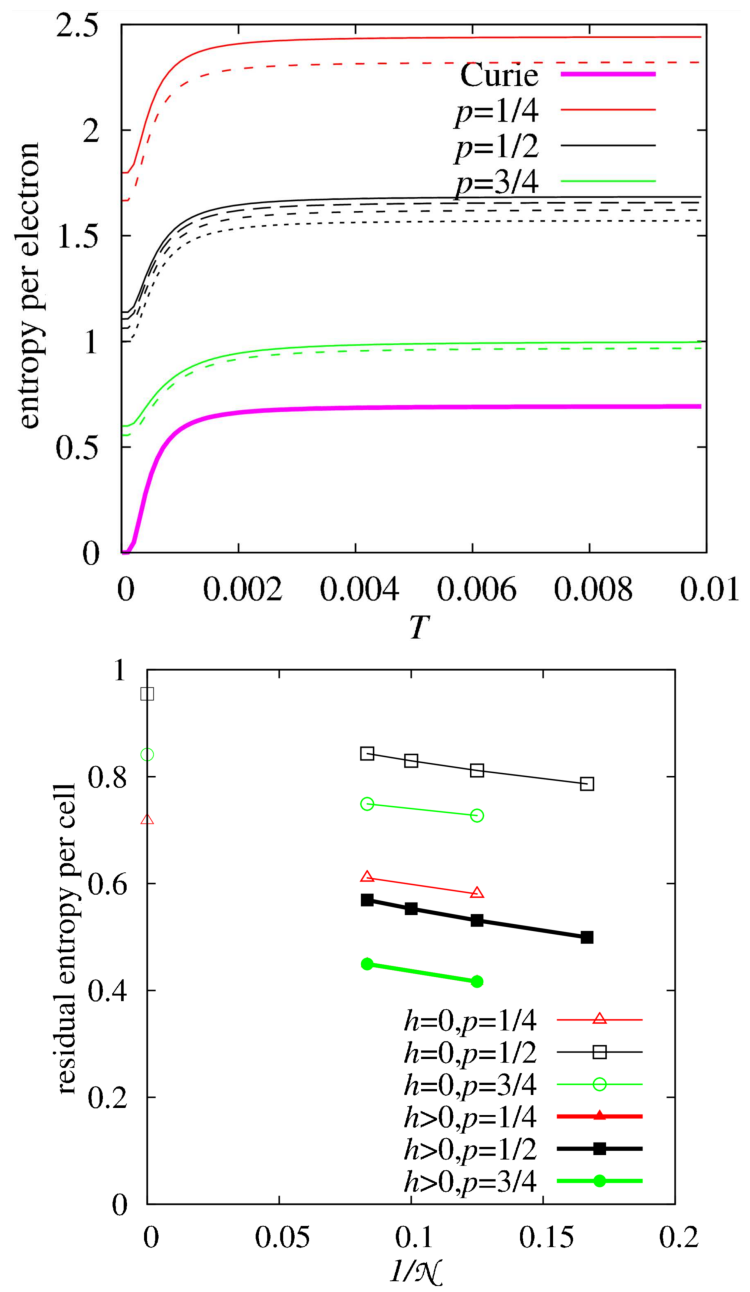

Fig. 2. Upper part: temperature dependence of the entropy per electron $s(T, h, p)$ for $h=0.001$ and $p=1 / 4$, $p=1 / 2$, and $p=3 / 4$. The results for $\mathcal{N}=6,8,10,12$ cells are shown by dotted, short-dashed, long-dashed, solid curves, respectively. The bold magenta curve corresponds to the Curie paramagnet. Lower part: dependence of the residual entropy per cell $S(T=$ $0, h, n, \mathcal{N}) / \mathcal{N}$ on $1 / \mathcal{N}$ for $h=0$ (thin curves) and $h>0$ (thick curves)for electron concentrations $p=1 / 4$, $p=1 / 2$, and $p=3 / 4$. Note that the thick red and green curves coincide. The results in the thermodynamic limit for $h=0$ are shown by symbols on the ordinate axis (i.e. at $1 / \mathcal{N}=0)$.

pronounced (peak becomes lower and broader) and $C(T, h, n, \mathcal{N}) \rightarrow 0$, when $p \rightarrow 1$. This indicates that less excited states are accessible in the Tasaki-Hubbard system.

The low-temperature magnetization curves in Fig. 4 show that it is easier to magnetize the Tasaki-Hubbard paramagnet with $p>0$ than the Curie paramagnet, i.e., the Tasaki-Hubbard paramagnet shares properties with a superparamagnet. This is in agreement with results for the initial (zero-field) susceptibility shown in Fig. 5.

Finally, we discuss the ground-state magnetic properties of the Tasaki-Hubbard paramagnet at $h=0$. To

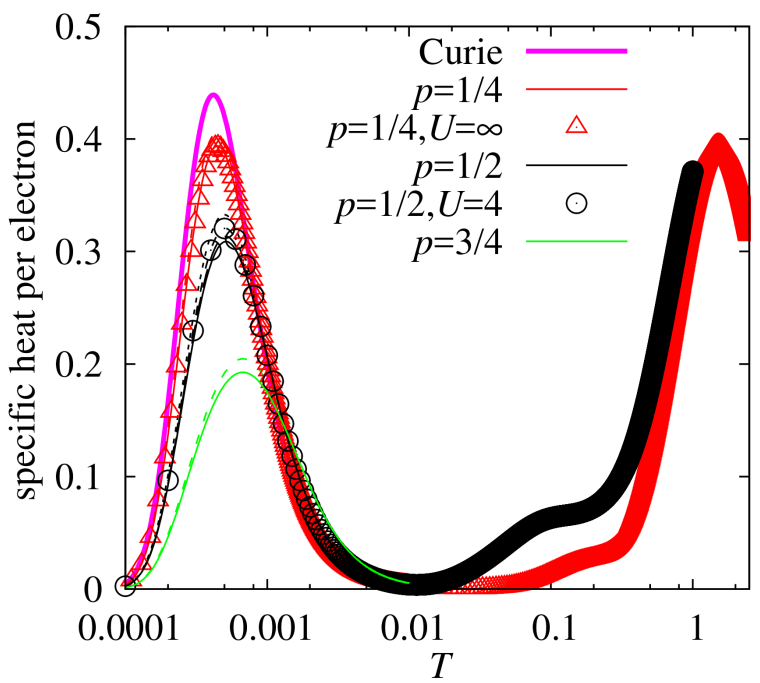

Fig. 3. Temperature dependence of the specific heat per electron $c(T, h, p)$ for $h=0.001$ and $p=1 / 4$, $p=1 / 2$, and $p=3 / 4$. The results for $\mathcal{N}=6,8,10,12$ cells are shown by dotted, short-dashed, long-dashed, solid curves, respectively. The bold magenta curve corresponds to the Curie paramagnet. By symbols we show exact-diagonalization data for the Hubbard model (1) with $U=4, N=16, n=4$ (०) and $U \rightarrow \infty, N=24$, $n=3(\Delta)$.

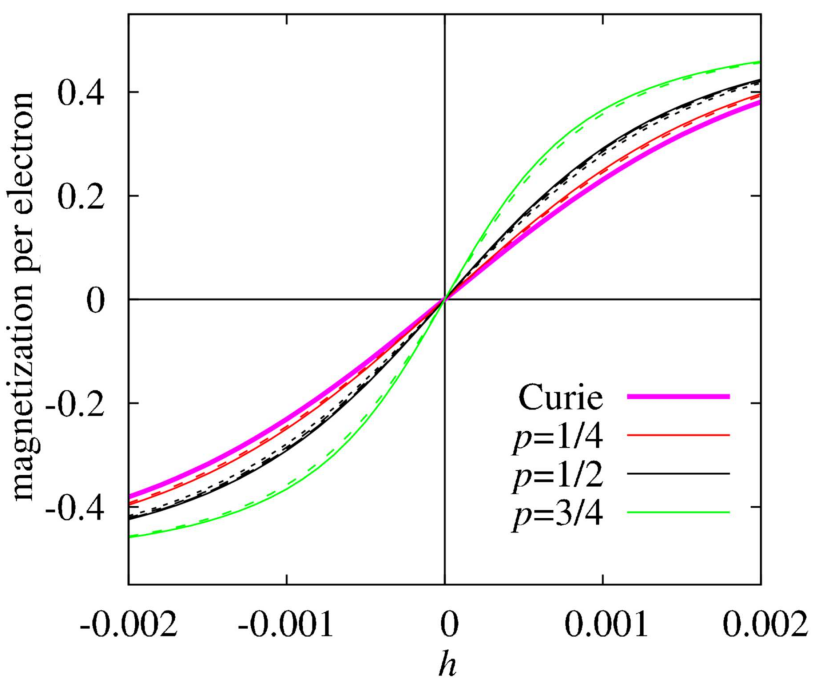

Fig. 4. Field dependence of the magnetization per electron $m(T, h, p)$ for $T=0.001$ and $p=1 / 4, p=1 / 2$, and $p=3 / 4$. The results for $\mathcal{N}=6,8,10,12$ cells are shown by dotted, short-dashed, long-dashed, solid curves, respectively. The bold magenta curve corresponds to the Curie paramagnet.

this end, we calculate the average square of the total spin $\left\langle\boldsymbol{S}^{2}\right\rangle_{n, \mathcal{N}}[7,8]$ The direct way to get $\left\langle\boldsymbol{S}^{2}\right\rangle_{n, \mathcal{N}}$ is to use its definition. For the considered example $n=4$, $\mathcal{N}=6$, in 30 states $\boldsymbol{S}^{2}$ has the value 6 , in 48 states $\boldsymbol{S}^{2}$ has the value $9 / 2$, and in 27 states $\boldsymbol{S}^{2}$ has the value 4 . Therefore $\left\langle\boldsymbol{S}^{2}\right\rangle_{4,6}=24 / 5$. An alternative way to get $\left\langle\boldsymbol{S}^{2}\right\rangle_{n, \mathcal{N}}$ is to calculate first the (normalized) number 


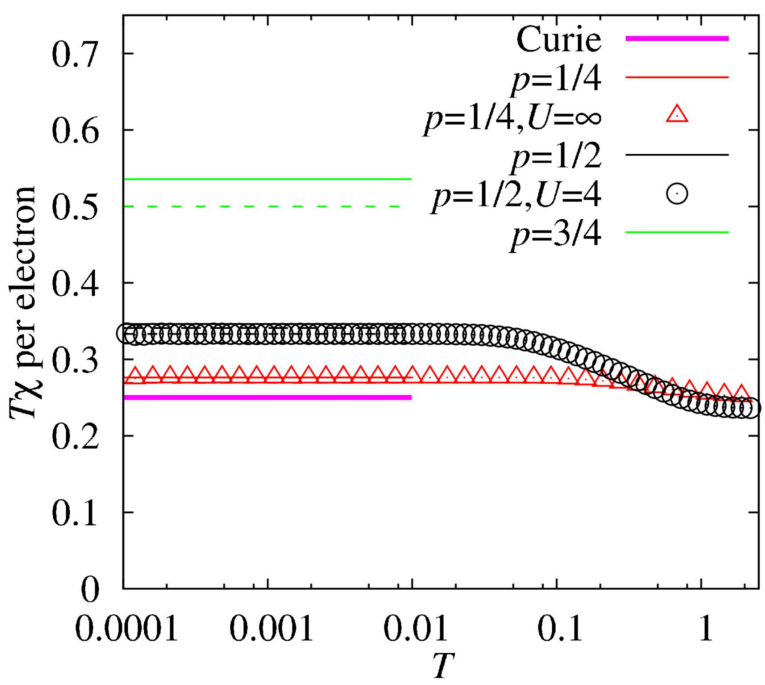

Fig. 5. Temperature dependence of the susceptibility per electron $\chi(T, h, p)$ multiplied by $T$ for $h=0$ and $p=1 / 4, p=1 / 2$, and $p=3 / 4$. The results for $\mathcal{N}=6,8,10,12$ cells are shown by dotted, shortdashed, long-dashed, solid curves, respectively. The bold magenta curve corresponds to the Curie paramagnet. By symbols we show exact-diagonalization data for the Hubbard model (1) with $U=4, N=16, n=4$ (०) and $U \rightarrow \infty, N=24, n=3(\Delta)$.

of clusters with $l$ electrons, $n(l)$, and then to sum up $\sum_{l=1}^{n} n(l)(l / 2)[(l / 2)+1]$, see Ref. [8]. For the considered example, $n(1)=48 / 105, n(2)=54 / 105, n(3)=48 / 105$, and $n(4)=30 / 105$ resulting again in $\left\langle\boldsymbol{S}^{2}\right\rangle_{4,6}=24 / 5$. The latter approach is convenient in the percolation setup, since the cluster-size distribution $n(l)$ can be obtained either analytically or numerically. Thus, in the one-dimensional case, $\left\langle\boldsymbol{S}^{2}\right\rangle_{n, \mathcal{N}} / \mathcal{N}=3 p(2-p) /[8(1-p)]$ in the thermodynamic limit [8]. A third possibility to get $\left\langle\boldsymbol{S}^{2}\right\rangle_{n, \mathcal{N}}$ is to exploit the Curie law for the temperature dependence of the initial susceptibility, $\chi(T, h=0)=$ $C / T$, since for Curie constant holds $C=\left\langle\boldsymbol{S}^{2}\right\rangle_{n, \mathcal{N}} / 3$. Using the latter approach we arrive at

$$
\left\langle\boldsymbol{S}^{2}\right\rangle_{n, \mathcal{N}}=\left.3 T^{2} \frac{\partial^{2} \ln Z(T, h, n, \mathcal{N})}{\partial h^{2}}\right|_{h=0} .
$$

In Table I we collect our findings for $\left\langle\boldsymbol{S}^{2}\right\rangle_{n, \mathcal{N}}, n=$ $1, \ldots, \mathcal{N}-1$ which coincide with the ones for $\mathcal{N}=6,8$ presented earlier in Ref. [7]. In Fig. 6 we show the results for $\left\langle\boldsymbol{S}^{2}\right\rangle_{n, \mathcal{N}}$ obtained by Eq. (8) demonstrating that (i) ferromagnetism disappears for $0<p<1$ as $\mathcal{N}$ increases (main part) and (ii) $\left\langle\boldsymbol{S}^{2}\right\rangle_{n, \mathcal{N}} / \mathcal{N}$ approaches $3 p(2-p) /[8(1-p)]$ (bold black curve) [8] as $\mathcal{N}$ increases (inset).

\section{Conclusions}

To summarize, in the present study we have extended the analysis of Refs. [6, 7] for the Tasaki-Hubbard chain. We switch on an infinitesimally small external magnetic field and show how previous findings become modified. At small electron densities the system is a

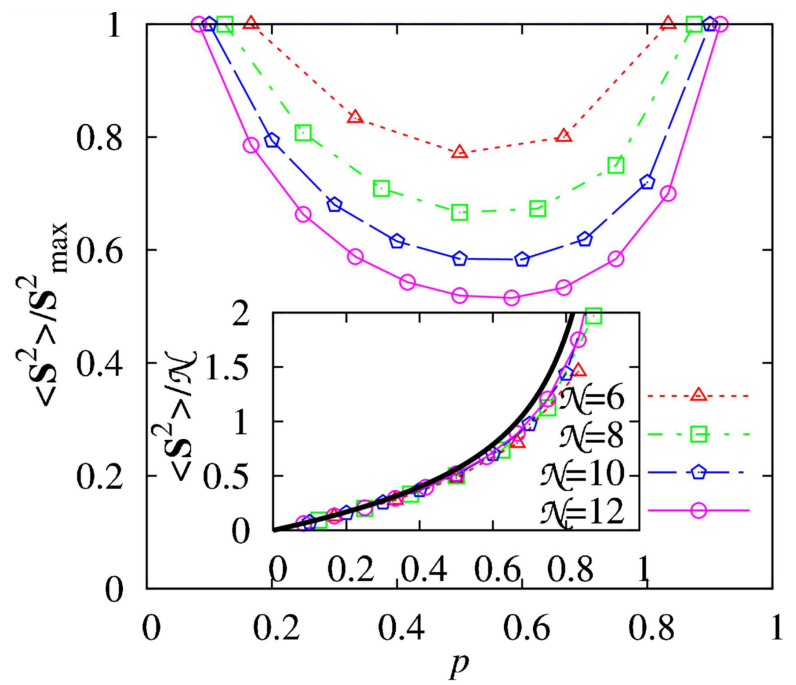

Fig. 6. Main part: dependence of the averaged (over ground states) total spin squared $\left\langle\boldsymbol{S}^{2}\right\rangle_{n, \mathcal{N}}$ per its maximal value $\boldsymbol{S}_{\max }^{2}=(n / 2)[(n / 2)+1]$ on the electron density $p$. The results for $\mathcal{N}=6,8,10,12$ cells are shown by dotted, short-dashed, long-dashed, solid curves, respectively. Inset: dependence of the averaged (over ground states) total spin squared $\left\langle\boldsymbol{S}^{2}\right\rangle_{n, \mathcal{N}}$ per cell on the electron density $p$. The bold black curve corresponds to the $\mathcal{N} \rightarrow \infty$ result obtained in Ref. [8], see the main text of the paper.

TABLE I

Values of $\left\langle\boldsymbol{S}^{2}\right\rangle_{n, \mathcal{N}}$ for Tasaki chains with different number of electrons and cells as they follow from Eq. (8).

\begin{tabular}{c|c|c|c|c|c|c|c|c|c|c|c}
\hline \hline$n$ & 1 & 2 & 3 & 4 & 5 & 6 & 7 & 8 & 9 & 10 & 11 \\
\hline $\mathcal{N}=6$ & $\frac{3}{4}$ & $\frac{5}{3}$ & $\frac{81}{28}$ & $\frac{24}{5}$ & $\frac{35}{4}$ & & & & & & \\
$\mathcal{N}=8$ & $\frac{3}{4}$ & $\frac{21}{13}$ & $\frac{117}{44}$ & 4 & $\frac{165}{28}$ & 9 & $\frac{63}{4}$ & & & & \\
$\mathcal{N}=10$ & $\frac{3}{4}$ & $\frac{27}{17}$ & $\frac{51}{20}$ & $\frac{48}{13}$ & $\frac{225}{44}$ & 7 & $\frac{39}{4}$ & $\frac{72}{5}$ & $\frac{99}{4}$ & & \\
$\mathcal{N}=12$ & $\frac{3}{4}$ & $\frac{11}{7}$ & $\frac{189}{76}$ & $\frac{60}{17}$ & $\frac{19}{4}$ & $\frac{81}{13}$ & $\frac{357}{44}$ & $\frac{32}{3}$ & $\frac{405}{28}$ & 21 & $\frac{143}{4}$
\end{tabular}

paramagnet. However, it differs from the conventional Curie paramagnet, rather it resembles a superparamagnet with a huge degeneracy of the ground state and a size-distribution of the ferromagnetic clusters. Comparison to exact-diagonalization data shows that the elaborated approach is appropriate for description of the low-temperature properties of the Tasaki-Hubbard-chain paramagnet. While with modest efforts the calculation of $Z(T, h, n, \mathcal{N})$ can be extended for larger $\mathcal{N}$, exactdiagonalization computations for $n=6(n=5)$ electrons and $\mathcal{N}=8(\mathcal{N}=12)$ cells if $U$ is finite (if $U \rightarrow \infty)$ are at the nowadays limits.

\section{Acknowledgments}

The present study was supported by the DFG (project RI615/21-1). O.D. acknowledges the kind hospitality of the University of Magdeburg in March-May of 2016. The work of O.D. was partially supported by Project FF-30F (No. 0116U001539) from the Ministry of Education and 
Science of Ukraine. O.D. would also like to thank the Abdus Salam International Centre for Theoretical Physics (Trieste, Italy) for partial support of this study through the Senior Associate award.

\section{References}

[1] H. Tasaki, Phys. Rev. Lett. 69, 1608 (1992).

[2] A. Mielke, J. Phys. A 24, L73 (1991); A. Mielke, J. Phys. A 24, 3311 (1991); A. Mielke, J. Phys. A 25, 4335 (1992); A. Mielke, Phys. Lett. A 174, 443 (1993).

[3] A. Mielke, H. Tasaki, Commun. Math. Phys. 158, 341 (1993).
[4] H. Tasaki, J. Phys. Condens. Matter 10, 4353 (1998); H. Tasaki, Prog. Theor. Phys. 99, 489 (1998).

[5] O. Derzhko, J. Richter, M. Maksymenko, Int. J. Mod. Phys. B 29, 1530007 (2015).

[6] O. Derzhko, A. Honecker, J. Richter, Phys. Rev. B 76, 220402(R) (2007).

[7] O. Derzhko, J. Richter, A. Honecker, M. Maksymenko, R. Moessner, Phys. Rev. B 81, 014421 (2010).

[8] M. Maksymenko, A. Honecker, R. Moessner, J. Richter, O. Derzhko, Phys. Rev. Lett. 109, 096404 (2012). 